\title{
VIOLENCIA OBSTÉTRICA. APORTES DESDE AMÉRICA
}

\section{LATINA}

\section{Patrizia Quattrocchi ${ }^{1}$}

Resumen: En América Latina, en la década pasada, se introdujo un nuevo término legal para proteger a las mujeres durante el parto: "violencia obstétrica" (VO). El concepto se refiere a actos en el contexto del parto y del nacimiento categorizados como violencia física o psicológica, debido al uso injustificado de intervenciones médicas, al trato deshumanizador y al abuso de patologización de los procesos naturales. Existen leyes específicas contra la violencia obstétrica en países como Venezuela (2007), Argentina (2009) y México (2014), Brazil (2017) y Uruguay (2017). En estos países la violencia obstétrica se considera un tipo de violencia basada en el género y una violación de los derechos humanos relacionados con la salud y con la salud reproductiva. En Europa, el tema es discutido por las organizaciones de los derechos humanos y los movimientos sociales, con el fin de luchar por un parto más humano y respetuoso, pero ningún país ha aprobado una legislación al respecto todavía. A pesar de la reciente implementación de Observatorios sobre violencia obstétrica en algunos países (Italia, España, Francia), el debate público y político sobre el tema es aún débil. El objetivo de esta ponencia es presentar los avances de investigación del proyecto "Obstetric Violence. The new goal for research, policies and human rights in childbirth". El proyecto tiene el objetivo de transferir - en términos de conocimientos - a los países europeos las experiencias aplicadas para el reconocimiento y la prevención de la VO en Latinoamérica, con el fin de proporcionar herramientas innovadoras con las cuales replantear la calidad de los servicios de atención al parto y al

\footnotetext{
${ }^{1}$ Doctora en Etno-Antropología en la Universidad de Roma "La Sapienza" (2005) con la tesis "Mujer maya y salud reproductiva en una comunidad maya de Yucatán. El caso de la sobada". Areas de investigación: antropología de la salud, antropología de la reproducción, salud reproductiva, salud intercultural, salud y género, políticas públicas en salud, metodologías cualitativas de investigación aplicadas a salud. Profesora investigadora en la Universidad de Udine (Italia).
} 
nacimiento; y proporcionar a la sociedad civil nuevos elementos para discutir el tema del parto. Los datos corresponden al trabajo de campo de corte etnográfico realizado durante el primer año en Argentina.

Palabras claves: Violencia obstétrica. Parto respetado. Parto y derechos humanos. Medicalización del parto

Abstract: In Latin America, over the past decade, the term "obstetric violence" (OV) has become part of the legal framework. The concept refers to acts in the context of labour and birth categorised as physically or psychologically violent due to unjustified use of medical interventions. Specific laws against obstetric violence a type of gender-based violence and violation of human rights - exist in Venezuela (2007), Argentina (2009) and Mexico (2014), Brazil (2017) y Uruguay (2017). In Europe, the issue is raised by human rights organisations and social movements fighting for more humane and respectful births. In Spain, Italy and France, observatories on $\mathrm{OV}$ were implemented, but no country has passed legislation on the matter yet. The concept is far from receiving sufficient critical examination within biomedical practice and public policy, and it is not seen as a potential tool for rethinking and improving birth care policies and practices. The purpose of this paper is to present the preliminary results of a biennial anthropological research "Obstetric Violence. The new goal for research, policies and human rights in childbirth". The project objective is transferring the Latin American experiences on recognising and preventing $\mathrm{OV}$ to the European context in order to provide decision makers with an innovative theoretical and methodological tool for rethinking the quality of birth care services and providing society with new ways to discuss childbirth issues. The data correspond to the field work performed in the first year in Argentina.

Key-words: Obstetric violence. Respectful maternity care. Human rights in childbirth. Medicalization of childbirth.

\section{Introducción}

El objetivo de este trabajo es presentar algunos avances del proyecto de investigación antropológica 
"Obstetric Violence. The new goal for research, policies and human rights in childbirth". Los datos corresponden al trabajo de campo realizado en Argentina, desde octubre de 2016 hasta marzo de $2017 .^{2}$

En las décadas pasadas, el embarazo y el parto se han vuelto cada vez más medicalizados en muchas partes del mundo. El parto es muy a menudo concebido y tratado como un evento médico, lo que requiere control, gestión de riesgos y seguimiento constante del cuerpo y la salud de la mujer (WHO 1985, 1996, 2008, 2015).

En muchos hospitales europeos, se ha introducido en las últimas décadas un modelo más humanizado de nacimiento, que incluye habitaciones hogareñas, posición libre durante el parto, uso de la bañera, etc. Sin embargo, en la mayoría de los países, el nacimiento todavía incluye una variedad de intervenciones médicas de rutina, tales como fármacos que inducen y aceleran el parto, posición litotómica obligatoria, maniobras invasivas, monitoreo fetal

\footnotetext{
${ }^{2}$ El proyecto es financiado por el Programa de Investigación e Innovación de la Comisión Europea Horizon 2020 (Marie SklodowskaCurie grant agreement No. 700946). La investigación se realiza en Argentina (2016-
}

continuo, episiotomía y un exceso de partos quirúrgicos (cesárea).

En muchos casos, estas intervenciones también se llevan a cabo en las mujeres con embarazos de bajo riesgo, sin un examen suficiente de los beneficios y riesgos para la madre y el bebé. Esto sucede a pesar de las recomendaciones de la Organización Mundial de la Salud (OMS) y las evidencias científicas, que desde hace más de cuatro décadas impulsan los gobiernos y las instituciones de salud para implementar programas para desmedicalizar el proceso reproductivo y disminuir las intervenciones innecesarias; entre estas la tasas de cesáreas (WHO 1985, 1996, 2015).

En 1996, en el documento Cuidado en el parto normal. Una guía práctica, la OMS declaró que "el objetivo de la atención es lograr una madre y un niño sanos con el mínimo nivel posible de intervención, compatible con la seguridad"; y que "en el parto normal, deberíamos tener una razón válida para interferir con el proceso natural". En el documento se

2017, Universidad Nacional de Lanus); y en Europa/Italia (2017-2018, Universidad de Udine). Aprovecho la ocasión para agradecer al director del Instituto Hugo Spinelli y a todos los integrantes del mismo. 
analizan 59 prácticas que se realizan de manera rutinaria en los hospitales. Las prácticas citadas se dividen en 4 categorías: a) prácticas que son claramente útiles y que debieran ser promovidas; b) prácticas que son claramente perjudiciales o ineficaces que debieran ser eliminadas; c) prácticas de las que no existe una clara evidencia para fomentarlas y que debieran usarse con cautela hasta que nuevos estudios clarifiquen el tema. Entre las prácticas perjudiciales o ineficaces que debieran ser eliminadas (categoría B), aparece el uso rutinario del enema y del rasurado púbico, la infusión intravenosa de rutina en el parto, la inserción de una cánula intravenosa profiláctica de rutina, la posición en decúbito supino de rutina durante la dilatación, el examen rectal, el uso de pelvimetrías, la administración de oxitócicos antes del nacimiento si sus efectos no pueden ser controlados, la posición rutinaria de litotomía con o sin estribos durante el parto, los esfuerzos de pujo sostenidos y dirigidos (maniobra de Valsalva) durante la segunda fase del parto, el masaje y estiramiento del periné durante la segunda fase del parto, el uso de tabletas orales de ergometrina en la tercera fase del parto para prevenir o controlar una hemorragia, el uso de ergometrina parenteral de rutina en la tercera fase del parto, el lavado rutinario del útero después del alumbramiento, y la revisión rutinaria manual del útero después del alumbramiento. Entre las prácticas que a menudo se utilizan inadecuadamente (categoría aparecen: la restricción de líquidos y alimentos durante el parto, el control del dolor con agentes sistémicos, el control del dolor con analgesia epidural, el monitoreo fetal electrónico, el uso de mascarilla y batas estériles durante la atención del parto, los exámenes vaginales repetidos 0 frecuentes (especialmente si los realiza más de un asistente), la estimulación con oxitocina, el cambio rutinario de la mujer de un lugar a otro al comenzar la segunda fase del parto, la cateterización de la vejiga, el animar a la mujer a pujar cuando la dilatación del cérvix es casi completa o ya completa y antes de que la mujer sienta imperiosa necesidad por sí misma, la rigidez de criterios acerca de la duración estipulada de la segunda fase del parto -como por ejemplo 1 hora-, incluso si las condiciones maternas y fetales son buenas y el parto progresa, las maniobras de alumbramiento, el uso 
liberal o sistemático de la episiotomía, y la exploración manual del útero después del alumbramiento. Se trata en total de 29 prácticas, que aún hoy en día y pese a las evidencias científicas y las directrices internacionales se siguen perpetuando en muchos hospitales tanto de los países desarrollados como de los países en desarrollo (WHO, 1996).

A veces las mujeres pueden sentirse obligadas para aceptar intervenciones médicas - sean de rutina o no -, o estas se llevan a cabo sin su consentimiento. En algunos casos, la coerción puede ser psicológica o implícita, es decir, relacionada con la autoridad del saber médico-científico y con las relaciones no horizontales entre médico-paciente (Lock e Nguyen, 2010). La autoridad médico-científica representa a veces una forma sutil para limitar el derecho fundamental de la mujer a participar activamente en el proceso mismo del parto y a decidir de manera libre y consciente sobre su cuerpo (Davis-Floyd e Sargent, 1997). El modelo médico hegemónico se convierte en este sentido en un conocimiento autorizado, "un poder que se da no porque es el correcto, sino porque es el que cuenta" (Jordan, 1997: 131).
En América Latina, en la década pasada, se introdujo un nuevo término legal para fomentar el ejercicio de los derechos reproductivos y para proteger a las mujeres contra la intervención innecesaria: "violencia obstétrica". El concepto se refiere a actos en el contexto del parto y del nacimiento categorizados como violencia física o psicológica, debido a la utilización injustificada de las intervenciones médicas.

Como veremos más adelante, además de ser considerada una violencia institucional y una violación de los derechos humanos relacionados con la salud y con la salud reproductiva, este tipo de violencia se considera basada en el género: una forma de violencia contra las mujeres dentro de un marco de subordinación y discriminación.

Hasta la fecha, los países que sancionaron leyes que contemplan la violencia obstétrica radican todos en América Latina: existen leyes específicas contra la violencia obstétrica en países como Venezuela (2007), Argentina (2009), México (2014), Brazil (2017) y Uruguay (2017). Venezuela fue el primer país en el mundo en sancionar una ley al respecto. En el artículo 15 de 
la Ley Orgánica sobre el Derecho de las Mujeres a una Vida Libre de Violencia," se define la violencia obstétrica como: "la apropiación de los procesos corporales y reproductivos de las mujeres por los profesionales de la salud, que se expresa como un tratamiento deshumanizado, un abuso de la intervención médica y la conversión de los procesos naturales en procesos patológicos, hecho que conlleva la pérdida de la autonomía y la capacidad de decidir libremente sobre su cuerpo y sexualidad, impactando negativamente en la calidad de vida". Según la definición venezolana, los siguientes actos ejecutados por el personal de salud se consideran violencia obstétrica: atención inoportuna e ineficaz a las emergencias obstétricas; obligación de dar a luz en una posición supina, con las piernas elevadas, cuando los medios necesarios para llevar a cabo un parto vertical están disponibles; impedir la unión temprana del niño con su madre sin una causa médica, bloqueando la posibilidad de contacto y lactancia materna después del nacimiento; alteración del proceso natural del parto de bajo riesgo mediante el uso de técnicas de aceleración, sin el consentimiento de la mujer; realización de un parto por cesárea cuando el parto natural es posible, sin el consentimiento de la mujer. Para los ejecutores de violencia obstétrica se prevén sanciones administrativas.

En México y - como veremos más adelante - en Argentina, la definición de violencia obstétrica es similar. En otros países, como Brasil, Chile, Ecuador, Perú, Colombia y Costa Rica el tema también está en discusión. En 2010, el Dr. Rogelio Pérez D'Gregorio, Presidente de la Sociedad de Obstetricia y Ginecología de Venezuela, mencionó el término "violencia obstétrica" en un editorial publicado en la Revista Internacional de Ginecología y Obstetricia, y el término entró también en el debate médico-científico internacional (Pérez D’Gregorio, 2010).

En Europa, el tema es discutido por las organizaciones de los derechos humanos (por ejemplo, Amnistía Internacional) y los movimientos sociales con el fin de luchar por un parto más humano y respetuoso, pero ningún país ha aprobado una legislación al respecto todavía. En algunos países, la intervención médica durante el parto se fue limitando, evitando las 
intervenciones que patologizan el proceso por ejemplo, los Países Bajos); pero en otros (por ejemplo, Italia y España), la medicalización del parto y del nacimiento es aún extendida. A pesar del interés creciente de la OMS (WHO, 2014), de Observatorios sobre violencia obstétrica en algunos países europeos (Italia, España, Francia y Grecia) y el activismo de las asociaciones civiles, el debate público y, sobre todo, político sobre el tema es aún débil. El concepto de "violencia obstétrica" está lejos de ser examinado en forma crítica dentro de la práctica biomédica y en el diseño/las discusiones de políticas públicas. El concepto es, en gran parte, desconocido por los profesionales de la salud o no se considera como una herramienta potencial para repensar la calidad de los servicios de atención durante el parto y la formación del personal de salud; en otras palabras, como una herramienta para contribuir al diseño de nuevas estrategias para disminuir la medicalización, especialmente en condiciones de embarazos fisiológicos.

Sin embargo, en Europa como en otros continentes, se reconoce ampliamente que el exceso de medicalización y la falta de respeto no benefician a las mujeres ni a sus hijos para obtener mejores resultados (WHO, 2014, 2015). Pero se priva a las mujeres de un papel activo en el parto. Como resultado, aumentan los costos de los sistemas de atención a la salud (por el uso de recursos innecesarios) y el número de controversias legales. Además, se limita a las mujeres el ejercicio de sus derechos de elegir las circunstancias de su parto, como fue establecido por la Corte Europea de los Derechos Humanos en el caso Ternovszky vs. Hungary en el $2010 .^{3}$

"En todo el mundo - ha declarado la OMS en el 2014 - que muchas mujeres sufren un trato irrespetuoso y ofensivo durante el parto en centros de salud, que no solo viola los derechos de las mujeres a una atención respetuosa, sino que también amenaza sus derechos a la vida, la salud, la integridad física y la no discriminación." El documento no menciona de manera explícita el concepto de "violencia obstétrica"; sin embargo, afirma que todas las mujeres tienen derecho a recibir el más alto nivel de cuidados en salud, que incluye el derecho a una atención

\footnotetext{
${ }^{3}$ Véase www.freedomforbirth.com
} 
digna y respetuosa en el embarazo y en el parto, y el derecho a no sufrir violencia ni discriminación. Y especifica: “el maltrato, la negligencia o la falta de respeto en el parto pueden constituirse en una violación de los derechos humanos fundamentales de las mujeres, descritos en las normas y los principios internacionales de derechos humanos." (OMS, 2014: 1)

\section{El proyecto de investigación sobre}

\section{violencia obstétrica}

En este contexto se desarrolla el proyecto de investigación "Obstetric Violence. The new goal for research, policies and human rights in childbirth". El objetivo general es la transferencia a los países europeos de las experiencias aplicadas para el reconocimiento y la prevención de la violencia obstétrica en Latinoamérica, con el fin de proporcionar herramientas teóricas $\mathrm{y}$ metodológicas innovadoras con las cuales replantear la calidad de los servicios de atención al parto y al nacimiento y fomentar un debate público sobre el tema. Los objetivos específicos son:
1) Analizar el proceso histórico, social y político que condujo al reconocimiento legal de la violencia obstétrica en algunos países de América Latina, concentrándose en Argentina.

2) Analizar el impacto que este reconocimiento ha tenido en los servicios de atención al parto y al nacimiento (según los datos oficiales) y en la formación de la próxima generación de profesionales de la salud (trabajo de campo) en Argentina.

3) Identificar y transferir elementos, buenas prácticas, herramientas y experiencias significativas con el fin de fomentar y apoyar un proceso de reconocimiento social y político de la violencia obstétrica en el contexto europeo, especialmente en países en los que la intervención médica durante el embarazo y durante el parto es alta, como en Italia y España. 
4) Diseñar e implementar un dispositivo (Plataforma de la violencia obstétrica), como espacio de información, consulta y asesoramiento para los tomadores de decisiones, para los investigadores y para quienes forman los profesionales de la salud ${ }^{4}$.

Los primeros dos objetivos se lograrán en Argentina durante el primer año de trabajo; los dos últimos en Italia/Europa durante el segundo año.

Con respeto a la aproximación teórica metodológica, la antropología médica - con contribuciones de disciplinas como la salud comunitaria, los derechos humanos y los estudios de género - proporciona el marco teórico interdisciplinario del proyecto. $\mathrm{La}$ metodología empleada es cualitativa y de corte etnográfico, cuyo propósito no es la simple descripción del fenómeno, sino su comprensión desde la perspectiva del actor social y desde un enfoque relacional (Menéndez, 2009). Los instrumentos y las técnicas utilizados son

${ }^{4}$ La Plataforma está en construcción el la página web del proyecto entrevistas semiestructuradas, abiertas y en profundidad con informantes clave (objetivo 1); la aplicación de una encuesta a estudiantes del último año del área de la salud y a residentes acerca de sus conocimientos de los contenidos de la ley sobre parto humanizado y de la ley que contempla la violencia obstétrica (objetivo 2); estudios de caso; compilación de un diario de campo, observación y observación participante.

El enfoque teóricometodológico está orientado a la solución de los problemas, y a aplicación de los resultados científicos para mejorar la realidad (Olivier de Sardan, 2005). En este caso específico, se trata de contribuir a mejorar las políticas y las prácticas relacionadas con el embarazo y el parto en el contexto europeo. La contribución será en términos de fomentar del debate público sobre el tema $y$ de recomendaciones para especialistas. Se redactará un "documento de recomendaciones" dirigido a los tomadores de decisiones y una "propuesta educativa" dirigida a los gestores de los procesos de formación

https://www.obstetricviolenceproject.com 
del personal de salud. El dispositivo implementado al final del proyecto (Plataforma) representará un instrumento de información, debate, consulta y asesoramiento; asimismo, permitirá el contacto entre los diferentes sectores y actores involucrados en el proceso político y formativo.

La visión general de esta investigación radica en la idea de que las experiencias en los países de América Latina - los únicos casos en el mundo hasta la fecha - constituyen un laboratorio innovador para el diseño, la implementación y el seguimiento de nuevas estrategias para unos mejores comprensión y manejo del parto y del nacimiento. En el contexto europeo, apoyar el reconocimiento social y político de la "violencia obstétrica" (analizando el concepto y sus potencialidades desde un enfoque crítico) significa fomentar una reflexión crítica sobre nuestras políticas y prácticas reproductivas, y brindar a las autoridades nuevas herramientas para limitar los excesos de medicalización, mejorar la calidad de los servicios de atención en el parto y la formación del personal de salud.
Los avances de investigación. El Observatorio de Violencia Obstétrica en Argentina

En este trabajo presentaré los avances de investigación que se han dado durante los primeros 6 meses de estancia en Argentina. En especial, quiero destacar una de las experiencias que estoy monitoreando en la ciudad de Buenos Aires, que desde el principio he identificado como un interesante "estudio de caso".

Se trata de la actividad de la Organización civil de corte feminista "Las Casildas", que se presenta a sí misma como una "una agrupación conformada por personas que, desde distintas áreas generamos dispositivos y aportes con el objetivo de difundir información en torno a la gestación, el parto, el nacimiento y la crianza de niños y niñas, como así también cuestiones de género" (www.lascasildas.com.ar).

En esta ocasión me quiero enfocar en el Observatorio de Violencia Obstétrica, surgido en el año 2014 como parte de la actividad de la Organización. En relación con los objetivos de mi proyecto, el Observatorio resulta ser una 
experiencia significativa en dos sentidos, que voy a tratar a continuación:

1) En sí mismo - como dispositivo político, social y cultural de concientización, visibilización y acción hacia un embarazo, un parto y un nacimiento libre de violencia;

2) Por sus posibles conexiones e interconexiones con experiencias similares, recién implementadas en algunos países europeos.

Intentaré profundizar lo que es el Observatorio, cómo nace, cómo funciona y cuáles son - desde mi punto de vista - su impacto y sus potencialidades a nivel político, cultural y social.

Los datos que presento surgen de 4 entrevistas en profundidad realizadas a las fundadoras del Observatorio y otros integrantes de Las Casildas; de 2 entrevistas a profesionales que - en algún momento - han colaborado con Las Casildas al respecto; de la observación y mi observación participante de 5 actividades de sensibilización/divulgación (talleres y charlas) sobre el tema de la violencia obstétrica y el trabajo del Observatorio; de la revisión del material escrito y visual elaborado, publicado o producido por las Casildas; del análisis de comunicados, reseñas y recortes de prensa al respecto; y de coloquios con mujeres que han participado en actividades realizadas por la Organización.

Antes de tratar del Observatorio, quiero mencionar brevemente el marco legal referente a la atención del embarazo, del parto y del nacimiento en Argentina. En el 2004 Argentina sancionó la Ley 25929, conocida también con el nombre de Ley de Parto Humanizado. La ley se reglamenta apenas en el 2015, con el decreto 2035/2015 de la Presidenta Cristina Khirchner. Esta Ley no menciona de manera directa a la violencia obstétrica; sin embargo declara que la mujer embarazada tiene derecho: a) a ser informada sobre las distintas intervenciones médicas que pudieren tener lugar durante esos procesos, de manera que pueda optar libremente cuando existieren diferentes alternativas. b) a ser tratada con respeto, y de modo individual y personalizado que le garantice la intimidad durante todo el proceso asistencial y tenga en 
consideración sus pautas culturales. c) A ser considerada, en su situación respecto del proceso de nacimiento, como persona sana, de modo que se facilite su participación como protagonista de su propio parto. d) Al parto natural, respetuoso de los tiempos biológico y psicológico, evitando prácticas invasivas y suministro de medicación que no estén justificados por el estado de salud de la parturienta o de la persona por nacer. e) A ser informada sobre la evolución de su parto, el estado de su hijo o hija y, en general, a que se le haga partícipe de las diferentes actuaciones de los profesionales. f) A no ser sometida a ningún examen o intervención cuyo propósito sea de investigación, salvo consentimiento manifestado por escrito bajo protocolo aprobado por el Comité de Bioética. g) A estar acompañada, por una persona de su confianza y elección durante el trabajo de parto, parto y postparto. h) A tener a su lado a su hijo o hija durante la permanencia en el establecimiento sanitario, siempre que el recién nacido no requiera de cuidados especiales. i) A ser informada, desde el embarazo, sobre los beneficios de la lactancia materna y recibir apoyo para amamantar. j) A recibir asesoramiento e información sobre los cuidados de sí misma y del niño o niña. k) A ser informada específicamente sobre los efectos adversos del tabaco, el alcohol y las drogas sobre el niño o niña y ella misma.

La ley define también el derecho del recién nacido a ser tratado en forma respetuosa y digna y a no ser sometido a ningún examen o intervención cuyo propósito sea de investigación o docencia, salvo consentimiento escrito. Además, se definen otros derechos del bebé: como el derecho a la internación conjunta con su madre en sala, y a que la misma sea lo más breve posible, teniendo en consideración su estado de salud y el de aquella. En fin, se mencionan los derechos de los padres a recibir información comprensible, suficiente y continua sobre el proceso o evolución de la salud de su hijo o hija, así como sobre los cuidados, el plan de vacunación, a tener acceso continuo a su hijo o hija mientras la situación clínica lo permita, a participar en su atención y en la toma de decisiones relacionadas con su asistencia, a que se facilite la lactancia materna. 
Otra ley que trata de los derechos de la mujer es la Ley 25673 de Creación del Programa Nacional de Salud Sexual y Procreación Responsable, reglamentada en el 2006, que tiene como objetivos alcanzar para la población el nivel más elevado de salud sexual y procreación responsable con el fin de que pueda adoptar decisiones libres de discriminación, coacciones o violencia; y a potenciar la participación femenina en la toma de decisiones relativas a su salud sexual y procreación. Tres años después, en el 2009, se sanciona y promulga también la Ley Nacional de Derechos del paciente con los profesionales e instituciones de salud 26529 que menciona - entre otras cosas - el trato digno y respetuoso, la intimidad, la confidencialidad, la autonomía de la voluntad y el consentimiento informado.

Además, en el año 2009 Argentina sanciona y promulga también la Ley 26485 o "Ley de protección integral para prevenir, sancionar $\mathrm{y}$ erradicar la violencia contra las mujeres en los ámbitos en que desarrollen sus relaciones interpersonales". En el art. 4 la ley define violencia contra las mujeres como "toda conducta, acción u omisión, que de manera directa o indirecta, tanto en el ámbito público como en el privado, basada en una relación desigual de poder, afecte su vida, libertad, dignidad, integridad física, psicológica, sexual, económica o patrimonial, como así también su seguridad personal. Quedan comprendidas las perpetradas desde el Estado o por sus agentes." Además, en el art. 5 se mencionan distintos tipos de violencia contra la mujer: la violencia física, psicológica, sexual, económica y patrimonial, y simbólica. El art. 6 define las formas en que se manifiestan los distintos tipos de violencia contra las mujeres en los diferentes ámbitos: violencia doméstica, violencia institucional, violencia laboral, violencia contra la libertad reproductiva, violencia mediática y violencia obstétrica.

Finalmente, la violencia obstétrica se define como "aquella que ejerce el personal de salud sobre el cuerpo y los procesos reproductivos de las mujeres, expresada en un trato deshumanizado, un abuso de medicalización y patologización de los procesos naturales, de conformidad con la Ley 25929.” La definición no incluye solo a los médicos, sino a todos los profesionales que ofrece atención a la 
mujer durante el embarazo, el parto y el nacimiento.

La definición legal de Argentina remite - como aquella venezolana y mexicana - a la desigualdad de género, que reproduce (y es producto) de la naturalización de la subordinación femenina a un sistema de organización social que desde el enfoque feminista se ha definido como "patriarcal", el cual involucra también los modelos de organización de los sistemas de salud de los Estados nacionales (Castro e Bronfam, 1993).

Sin embargo, la definición legal - y el lenguaje jurídico que la sustenta toma en cuenta solo en parte la complejidad de la violencia obstétrica y no permite comprender y explicar las raíces históricas, sociales, políticas y económicas que la producen y legitiman.

Desde una mirada socioantropológica, podemos en cambio situar la violencia obstétrica dentro de su contexto socio-histórico y desentrañar los múltiples aspectos y las tantas dimensiones que a menudo aparecen cuando hablamos de este tipo de violencia. Además, desde la misma

5 Castro define el curriculum oculto como el conjunto de reglas, conminaciones, valores entendidos, estrategias de adapatación, perspectiva podemos problematizar las distintas definiciones que se han dado de esta violencia y entender las lógicas, las trayectorias y el grado de apropiacion y/o de rechazo por parte de los distintos actores sociales involucrados, tanto a nivel individual como institucional (mujeres y hombres, profesionales de la salud, funcionarios, políticos, investigadores, instituciones, colectivos, asociaciones, etc.). La construcción del concepto de violencia obstétrica se vuelve en este sentido un "campo de disputa" (Castrillo, 2016), en donde los distintos sujetos interactúan y en donde diferentes estrategias de legitimación o deslegitimación de esta violencia son puestas en marcha, desde la perspectiva y el interés de cada uno.

Como muestra la creciente investigación sobre el tema, en esta arena se destacan - entre otros aspectos - las relaciones de poder entre médicos $\mathrm{y}$ pacientes, basadas en quién supuestamente tiene el saber; el tema de la formación médica, tanto en el currículo académico como en el currículo oculto $;{ }^{5}$ la organización de los servicios de salud; las variables de clase,

interiorizaciones y prácticas que se aprende en forma paralela al curriculum oficial (Castro, 2015). 
etnia y género como determinantes sociales de la salud y la mercantilización del proceso embarazo, parto $\mathrm{y}$ nacimiento y del producto-recién nacido.

Se trata, entonces, de un problema de origen social: de cómo se producen y reproducen las relaciones sociales y de poder entre individuos y entre individuos e instituciones; de cómo estas relaciones producen a su vez estructuras sociales e ideologías que se vuelven portadoras de "conocimientos autorizados", legitimados socialmente, no cuestionables y no cuestionados (como de hecho se percibe y se maneja el conocimiento biomédico o el "poder obstétrico”) (Arguedas Ramirez, 2014); de cómo este "conocimiento autorizado" surge y al mismo tiempo conlleva al desplazamiento y a la desvaloriziación sistématica de otros saberes, in primis el saber femenino (Camacaro Cuevas , 2012; Terán et alt., 2013; Bellón, 2015; GIRE, 2015); de cómo se dan en términos bourdeanos las relaciones entre campo médico y habito médico y de cómo se construye la subjetividad del médico (Castro, 2015); y de cómo - en fin - se da el entrelazamiento, entre

\footnotetext{
6 Véase - entre otras - la Convención sobre la
} eliminación de todas las formas de algunos o todos estos elementos, para no permitir en la práctica de atención cotidiana el ejercicio de los derechos reproductivos por parte de las mujeres, consideradas pacientes o usuarias de los servicios de salud, pero no concebidas y tratadas como personas (la mayoría de las veces sanas) sujetos de derecho (Castro e Erviti, 2003; Belli, 2013).

Para enfrentar estos procesos se propone hoy en día ampliar la mirada, abarcando el enfoque de los derechos humanos. Los derechos humanos en general (derechos a la integridad personal, a la privacidad e intimidad, a la información y a la autonomía y libertad en las decisiones, a un trato respetuoso, a no ser discriminados, entre otros) se entrelazan - desde una mirada integral con los derechos relacionados con el género, con la salud y con la salud reproductiva mencionados en las distintas convenciones y documentos internacionales. ${ }^{6}$ Todos estos derechos constituyen desde luego un eje transversal para comprender los procesos arriba mencionados en términos de "violación", de violencia estructural de género, de violencia Convención de Belén du Pará (1994). 
institucional y de reproducción-en sus distintos niveles - de desigualdad, de inequidad e injusticia (Sadler et al., 2016, Magnone, 2010; Castro, 2015; Belli, 2013).

Afirmar que parir sin violencia es un derecho humano y un derecho a la salud reproductiva que todas las mujeres poseen y pueden/deben ejercer sea cual fuera su origen, cultura, estado físico y psicológico, trayectoria, etc., permite insertar el tema de la violencia obstétrica en una perspectiva transcultural, estructural y relacional: la violencia obstétrica no es solo un problema de la mujer o del Estado; tampoco resulta ser solo un problema de calidad $\mathrm{y}$ de organización de los servicios de salud o de capacitación de los profesionales, como se ha manejado el asunto hasta tiempos recientes. Se trata de una cuestión más amplia que requiere de distintas perspectivas de análisis y de distintas herramientas de acción, algunas de las cuales deben ser propiciadas por el Estado (como la ley), otras que surgen desde la sociedad, como la militancia de grupos y asociaciones que defienden y reclaman los derechos reproductivos.

Género, poder y derechos humanos en el parto y el nacimiento son ejes de discusión y análisis también en la actividad de "Las Casildas", la Organización que en octubre de 2015 lanzó el Observatorio de Violencia Obstétrica en Argentina.

Los objetivos del Observatorio son bien definidos: recolectar y sistematizar datos y estadísticas relacionados con la violencia obstétrica, realizar seguimiento a denuncias, monitorear políticas públicas, proyectos de ley y cumplimiento de leyes, elaborar recomendaciones a organismos e instituciones involucrados, crear espacios de difusión y debate con profesionales y ciudadanía en general, promover la elaboración de estudios y proyectos de investigación, apoyar y difundir a acciones de otros colectivos y agrupaciones en torno a la violencia obstétrica y articular trabajo y acciones con otros Observatorios nacionales e internacionales. $^{?}$

Las fundadoras son dos integrantes de la organización, animadas por la idea de construir una red nacional que permitiera, a través de distintos

\footnotetext{
${ }^{7}$ www.lascasildas.com.ar., página consultada el 30 de marzo de 2017.
} 
dispositivos, visibilizar este tipo de violencia en el país.

Realmente, todo el mundo nos dijo “Están locas! ¡No van a poder! Esto nos dio más impulso, y el Observatorio lo lanzamos en octubre de 2015. Lo lanzamos muy a nuestro estilo, ya con 1000 mujeres encuestadas, la herramienta ya puesta en práctica en el territorio. No es que lo lanzamos $\mathrm{y}:$ ¿Y ahora que hacemos? No, ya veníamos trabajando con encuesta de atención al parto y/o a la cesárea, que la implementamos en el territorio a través de dos vías: una vía web y otra personalmente. (Julieta, 29 de enero de 2017)

Una de las primeras acciones relacionadas con el Observatorio fue como explica la fundadora - diseñar una encuesta de atención al parto y/o a la cesárea y subirla a la red, para que cada mujer del país pudiera recopilarla y enviarla a la Organización. El instrumento utilizado prevé 55 preguntas cerradas (posibles respuestas: sí, no, no recuerdo) o semiabiertas (especificar algo con más detalles) relacionadas con el trato recibido, con las prácticas realizadas hacia el cuerpo de la mujer y en el recién nacido, y con la información otorgada por parte de los profesionales de la salud. Los temas explorados son amplios y refieren al contenido de la ley
25929: presencia de acompañante, comentarios, chistes, sobrenombres, críticas contra la mujer, discriminación sufrida, intimidación (por ejemplo, a través de frases como "estás poniendo en riesgo la vida de tu bebé"), rasurado de genitales, enema, rotura artificial de bolsa, colocación de vía entravenosa, administración de oxitocina sintética, tactos vaginales, monitoreo fetal continuo, episiotomía, maniobra de Kristeller, inducción, anestesia, fórceps, posición del parto, cesárea, corte del cordón umbilical, contacto inicial y apego, lactancia, prácticas realizadas al bebé. Ejemplos de preguntas son:¿Estuviste acompañada por una persona de tu elección durante el trabajo de parto $^{8}$ O la pregunta n. 30 relacionada con la maniobra Kristeller (explicada entre paréntesis en el cuestionario) con posibilidad de contestar sí, no, no recuerdo. En caso de respuesta afirmativa (me la realizaron), se le pide a la mujer que conteste la siguiente pregunta: ¿Pidieron tu autorización para realizar esta práctica, informándote el porqué, sus riesgos y beneficios? Las respuestas previstas: sí, no, no recuerdo.

\footnotetext{
${ }^{8}$ Pregunta n. 9.
} 
La encuesta se lanzó a nivel nacional junto a la implementación del Observatorio, a través de la página internet de Las Casildas y de los medios de comunicación. Para llevarla a cabo se armó una red de encuestadoras que aplicaron el instrumento también de manera presencial en varias provincias de la Argentina. La recolección se realizó desde septiembre de 2015 hasta agosto de 2016. Se realizaron 4939 encuestas. El fin fue contar con datos cuantitativos que pudieran visibilizar el problema y llamar la atención de las instituciones públicas y de los tomadores de decisiones.

La idea era visibilizar a las mujeres de todos los estratos sociales, no solo aquella que pueden pagar su obra social o prepaga. Eso salió. (Julieta)

Entrevistamos a mujeres cartoneras, estuvimos en villa miseria, estuvimos en los centros de atención primaria, y entrevistamos a personas de clase medio-alta (Ester, 30 de marzo de 2017)

En octubre del 2016 los datos fueron presentados en el Encuentro Nacional de Mujeres que tuvo lugar en

\footnotetext{
${ }^{9}$ Las mujeres encuestadas tienen una edad media de 27,8 años; 38, 7 semana media de parto; $27,9 \%$ atendidas por el servicio público, 50, 4 por
}

Rosario. Por primera vez, el país contó con datos relacionados con lo que la ley 26485 define como "violencia obstétrica".

El incumplimiento de la ley de parto respetado es evidente en los datos presentados. Por ejemplo: a 3 de cada 10 mujeres $^{9}$ no se les garantizó su derecho de estar acompañadas durante el trabajo de parto; 4 de cada 10 no estuvieron acompañadas durante el parto/cesárea, 4 de cada 10 mujeres no pudieron moverse libremente durante el trabajo de parto, 7 de cada 10 no tuvieron libertad de movimiento durante el parto, 6 de cada 10 mujeres tuvo monitoreo fetal continuo, a 7 de cada 10 les pusieron una vía (conectada a un suero, no intermitente), 6.4 de cada 10 no pudieron beber o comer durante el trabajo de parto, a 7 de cada 10 mujeres les rompieron artificialmente la bolsa, a 6.4 de cada 10 mujeres les fue suministrada oxitocina para conducir y/o acelerar el trabajo de parto, a 3.3 de cada 10 mujeres les realizaron la maniobra Kristeller en su parto, a 6 de cada 10 mujeres se les practicó la episiotomía

el servicio privado, $17 \%$ por la obra social y $3 \%$ en el domicilio de la persona gestante. 
y, de estas, 8.5 de cada 10 eran primerizas. Además, los datos muestran que 4.7 de cada 10 nacimientos fueron por cesárea $\mathrm{y}$, de estas, 4 de cada 10 fueron programadas. A su vez, 8,8 de cada 10 cesáreas fueron realizadas a primerizas; 6,6 de cada 10 recién nacidos tuvieron contacto inmediato con su madre, pero en 6.3 de cada 10 casos ese contacto solo duró un momento. El informe destaca también la falta de información otorgada a la mujer durante los distintos momentos (Las Casildas, 2015).

El Observatorio explica estos datos por la presencia en el país de un modelo de atención al parto medicalizado e intervencionista, situación comprobada también por las instituciones de salud y por los organismos internacionales. De acuerdo con el Segundo Informe Nacional de Relevamiento Epidemiológico SIPGestión, la tasa de cesáreas en Argentina se ubica en un promedio de $30,6 \%$ de los partos en hospitales públicos registrados en el país entre 2010 y 2013, y entre el 60 y $70 \%$ en el sector privado (Ministerio de Salud de la Nación e Organización Panamericana de la Salud, 2015). Recordamos que la tasa
38 recomendable por la OMS desde las Recomendaciones de Fortaleza se sitúa entre 10 y $15 \%$, y que la reciente revisión sistemática de la misma institución internacional a nivel de población señala que las tasas de cesárea superiores al 10\% no están asociadas con una reducción en las tasas de mortalidad materna y neonatal (OMS, 2015). Recordemos, en fin, que la tasa de cesárea se considera a nivel internacional un indicador de idoneidad en la atención al parto.

Algunos hospitales y maternidades públicas adoptaron en sus protocolos ciertas prácticas que refieren a un modelo de atención "humanizado" (presencia de un acompañante, posición libre etc.). Pero la mayoría de los partos en Argentina siguen siendo muy intervenidos. Entre otras prácticas estandarizadas y rutinarias que se siguen llevando a cabo, cabe mencionar: episiotomías, aceleración o inducción del parto, maniobras, tactos vaginales múltiples, posición litótomica obligatoria, imposibilidad de moverse y de comer durante el trabajo de parto, rotura artificial de bolsa, cesárea programada. Estas prácticas se mantienen a pesar de las 
recomendaciones a nivel legislativo y a pesar de las evidencias científicas internacionales que las desaconsejan (WHO, 1996). Se trata de una situación denunciada de manera creciente a partir de la década pasada (Chiarotti et. alt., 2003; Chiarotti et alt., 2008).

Se trata de la perpetuación de un modelo hegemónico que no dialoga con las evidencias científicas, ni con las directrices internacionales y nacionales, y que omite las experiencias y las buenas prácticas de otros países. A su vez, este modelo tampoco dialoga con perspectivas que interpretan y manejan el parto y el nacimiento de un modo diferente, como por ejemplo el modelo no medicalizado que sustenta el parto a domicilio. Al contrario, es un modelo que se autosustenta y se autolegitimiza a través de dinámicas de poder y de biopoder: en la definición faucoultiana el poder del Estado sobre los cuerpos de los individuos (Faucoult, 1969, 1975). En este sentido y a través de dispositivos más o menos coercitivos, las mujeres embarazadas y las parturientas se vuelven cuerpos dóciles, controlados, hasta "moldeados" por la institución y por su ideal: cuerpos-objetos anestesiados, parados y mutilados (real o
39

simbólicamente), objetos de intervención. La dimensión subjetiva del "ser" y del "poder ser" desaparece en el proceso de naturalización y mistificación de la necesidad del "hacer" $y$, a menudo, del "hacerse hacer" por parte del profesional, el único legitimado a manejar el proceso.

El Observatorio y los resultados de la encuesta se han presentado en charlas informativas, talleres $\mathrm{y}$ conferencias. En las ocasiones en las cuales he participado, la presentación ha sido parte de un evento más amplio, que contempla el uso de otro instrumento, diseñado e implementado por Las Casildas: la obra de teatro "Parir(NOS)". En 45 minutos, 4 actrices relatan 4 experiencias de partos distintos: desde un parto por cesárea hasta un parto a domicilio, pasando por un parto vaginal muy intervenido y un parto respetado. En este marco, se exploran distintos modelos de atención. Lo que tienen en común estas experiencias es el grito dramático de las protagonistas: "Algo no está bien". El objetivo de la obra es visibilizar la violencia obstétrica a través de la narración y fomentar un debate público sobre el tema, para que las mujeres puedan conocer $\mathrm{y}$, sobretodo, re- 
conocer este tipo de violencia, de la que muchas veces han sido víctimas silenciosas.

Uno de los temas puestos en la mesa por Las Casildas es, de hecho, la naturalización de la violencia obstétrica, hecho que no permite el desarrollo de una concientización por parte de las mujeres, de sus parejas y de la sociedad en su conjunto. En este contexto, la obra de teatro se concibe como un "disparador" (en las palabras de la coautora del texto Violeta, que actúa en el escenario también) para llamar la atención tanto de personas que ya conocen o están involucradas en el tema, como de personas que se acercan a este tipo de violencia por primera vez:

Hay mujeres que van a la obra y lo primero que sucede es que reconocen por primera vez que hay violencia; o sea, se puede nombrar. Eso, poder por primera vez nombrar. Ya viste que en la obra repetimos mucho: “¡Algo no está bien!". Que es como el primer punto, es algo que te hace ruido, ese un "run run" interno que no logras ponerle nombre. (...) Eso que me pasó es violencia, se llama violencia obstétrica y se expresa de esta forma (Violeta, 22 de noviembre de 2016)

La reflexión que la obra provoca abarca también a los profesionales de la salud, que tampoco en su trayectoria profesional - tienen muchas ocasiones de desarrollar un sentido crítico hacias sus propias prácticas, conocimientos, actitudes, hábitos y protocolos:

También nos pasó con
profesionales, algunos
profesionalees al terminar la obra:
"bueno, yo nunca me había dado
cuenta que eso que yo hacía podía
generar eso. Reconocer esto, la
práctica violenta. Nunca se me
ocurrió. (Violeta)

Y no menos importante en la narrativa de la coautora de Parir(NOS), es el comentario al respecto de las parejas de las mujeres que sufrieron algún tipo de violencia, y que subraya las múltiples consecuencias que la violencia obstétrica tiene a mediano y a largo plazo, tanto a nivel individual como social:

Nos ha pasado también con compañeros que te dicen: "Claro, ahora la entiendo, cuando ella me hablaba de esto que había vivido, era como...dale...y de pronto verlo...ah claro es esto, esto es como ella se sintió". (..) Porqué no es lo mismo atravesar una experiencia tan intensa desde el placer, desde la autonomía, desde el reconocimiento del cuerpo, y para este compañero acompañar esto, que acompañar una escena de violación. $\mathrm{O}$ sea, ¿cómo me encuentro en la intimidad después que yo he sido violada y tu estabas allí? Más allá de lo fisiológico y de lo hormonal, la experiencia del parto obviamente se juega en la intimidad de esta pareja". Para ellos también...el poder verlo, 
poder reconocerlo, poder decir "fue horrible". (Violeta)

La eficacia de la obra es justamente esta: dar voz a las tantas mujeres que no tienen o no han tenido voz para relatar su experiencia de sufrimiento, dejando fluir o reaflorar sensaciones $\quad \mathrm{y} / \mathrm{o} \quad$ emociones experimentadas, y jamás resignificadas y/o reelaboradas. La incorporación del sufrimiento expresado a través de las palabras, la corporalidad, y de los gestos de las actrices permite desarrollar sentimientos de identificación y empatía, o cuando menos dudas y curiosidad. El "disparador" actúa de hecho no sólo hacia las mujeres, sino hacia todos los que asisten a la actuación. El debate posterior - que durante mi participación en los eventos siempre se ha dado de manera espontánea a partir de preguntas o del relato de experiencias vividas por parte de las mujeres- permite compartir reflexiones entre los asistentes, desarrollando un proceso de aprendizaje empático que conlleva a un proceso de

\footnotetext{
${ }^{10}$ Cabe señalar que el trabajo de la organización contempla otras actividades. Entre éstas, la organización de talleres dirigidas a mujeres, a profesionales de la salud y a la ciudadanía en general; charlas informativas sobre el tema de los
}

introyección de la información a través de la emoción y la identificación.

Cabe destacar que la obra segun las integrantes de las Casildas representa un valor agregado en sí mismo, por haber permitido presentar y discutir el tema de la violencia obstétrica en lugares distintos (dentro y fuera de Argentina) y en espacios a veces difíciles de alcanzar para una Organización civil:

Todos los meses tenemos, por lo menos, una función. Hemos estado en instituciones. Hemos estado en hospitales, en la facultad de medicina ya estuvimos dos veces, en otras facultades, de enfermería, medicina, psicología. Viajamos a Paraguay, viajamos a Chile con la obra, en salitas de atención primaria, en distintos lugares. Nos han permitido entrar a lugares en donde con una charla no...no íbamos a entrar, a un hospital no nos iban a invitar a hablar a nosotras. Jamás. (Violeta)

\section{A modo de cierre}

El "formato" propuesto por las Casildas (obra de teatro, observatorio y debate $)^{10}$ tiene impacto en distintos ámbitos y por distintas motivaciones. Antes que nada, se presenta como un instrumento de información y sensibilización que puede interesar a

derechos sexuales y reproductivos, parto respetado, violencia; organización de eventos de sensibilización sobre estas temáticas, ideación, producción o co-producción de materiales audiovisuales, documentales, spots, artículos de prensa, informes, publicaciones. 
personas de diferentes sectores sociales, diferentes trayectorias de vida $y$ experiencias. De hecho, como señalamos, se presentó en distintas ocasiones, tanto entre especialistas (hospitales, centros de salud, universidades, instituciones) como en espacios de debate públicos dirigidos a toda la población.

Más allá de su difusión durante la actuación de la obra teatral, la relevancia del Observatorio de violencia obstétrica se encuentra, desde mi punto de vista, en distintos niveles, relacionados entre sí. Se trata - de lo que he podido comprender hasta la fecha, por lo menos de los siguientes aspectos:

- Como dispositivo social, el Observatorio permite dar visibilidad a un tema aún poco debatido (cuando no desconocido) entre los especialistas y la ciudadanía. Esto contribuye a crear una conciencia social con respeto a este tema, otorgándole la importancia que merece, en relación con uno de los momentos básicos de la vida individual y colectiva; un evento “elemental" diría Marc Augé (1986): el nacimiento.

- Como dispositivo cultural, el Observatorio permite construir y difundir un discurso crítico hacia los mecánismos de construcción y legitimación de las relaciones de poder entre mujeres $y$ hombres, y entre individuos e instituciones. Además, permite abordar temas relacionados con la concepción del parto y del nacimiento (proceso normal vs. acto/evento médico), con la autoridad del conocimiento médico y obstétrico y con el significado cultural y simbólico de ciertas prácticas relacionadas con el parto hospitalario. ${ }^{11} \mathrm{Y}$, no menos importante, permite reconocer y desnaturalizar situaciones de violencia presentes en la vida cotidiana, tanto contra las mujeres (violencia de género en sus distintas formas) como contra la ciudadanía en general (violencia

11 Véase al respeto el largo trabajo de DavisFloyd sobre los rituales hospitalarios en Estados Unidos. 
institucional y violación de los derechos humanos, entre otras).

- Como dispositivo político. Desde el punto de vista político, el Observatorio constituye un instrumento para canalizar los datos recolectados y elaborados (por ejemplos, estadísticas) y acercarlos a los tomadores de decisiones. Este es un punto importante: como sabemos, la implementación de cualquier política pública se da a través de una clara identificación y cuantificación del problema. Si bien es verdad que el dato estadístico no es suficiente para dar cuenta de las experiencias subjetivas de los actores involucrados en un proceso social (en este caso de las mujeres, pero podría ser de los varones o de los profesionales de la salud), es importante contar con información de este tipo para poder empezar a construir un diálogo con las instituciones promotoras de políticas públicas

12 Con respeto a la violencia obstétrica Las Casildas ofrece también asesoría acompañamiento en caso de denuncia. en salud. Desde el punto de vista político, el Observatorio es también un dispositivo de participación ciudadana, en cuanto instrumento implementado por una Organización civil y como espacio a través del cual los ciudadanos en general (mujeres, pero no solo ellas) pueden conocer, afirmar y ejercer sus derechos. ${ }^{12}$

- Como dispositivo transcultural y transdisciplinario. Desde este punto de vista, me parece muy interesante el papel que el Observatorio está desempeñando a nivel internacional, como "formato" inspirador de experiencias similares implementadas o en vía de implementación en otros países. Tanto en América Latina (Chile, Uruguay) como en Europa (Italia, España, Francia, Grecia) se reconoce la experiencia argentina como referente $^{13}, y$ como Organización líder en la

\footnotetext{
${ }^{13}$ Se trata de un tema que profundizaré - sobre todo con respeto a los países europeos - a mi regreso en Italia.
} 
creación de Inter-OVO-Red Internacional de Observatorios de Violencia Obstétrica, instrumento de diálogo entre los distintos Observatorios que ya existen en el mundo. ${ }^{14}$

Hace falta mencionar que el Observatorio de Violencia Obstétrica podría volverse también un dispositivo pedagógico en el aprendizaje social (de los ciudadanos en general) y médicoobstétrico. Con respecto a este punto, si el concepto de violencia obstétrica en sus distintas formas y distintos significados fuese realmente aceptado por la comunidad médico-obstétrica como un instrumento crítico para ampliar la propia visión del proceso reproductivo y mejorar las propias prácticas, podríamos considerar al Observatorio como un escenario de aprendizaje, que a partir de los datos concretos, contribuye a plantear caminos innovadores de formación/actualización del personal de salud. Mientras, sin dudas, dar a conocer al Observatorio entre los profesionales y los formadores del área de la salud,

14 Véase la Declaración conjunta de los Observatorios de Violencia Obsétrica de Argentina, Chile, Colombia, España y Francia (8 de marzo de 2016, puede aportar en términos de formación y sensibilización.

Cabe señalar, por ende, que todas las dimensiones arriba mencionadas (social, cultural, política, pedagógica) están entrelazadas en la construcción de un interesante metadiscurso crítico - que queda por explorar por mi parte - sobre cómo queremos ser, cómo individuos y sociedad.

\section{Bibliografia}

Arguedas Ramírez, Gabriela (2014), "Violencia Obstétrica. Propuesta conceptual a partir de la experiencia costarricense," Cuadernos Intercambio sobre Centroamérica y el Caribe, 11(1), 1659-4940.

Augé, Marc; Herzlich, Claudine (1986), Il senso del male: antropologia, storia e sociologia della malattia. Trad. Annick Wouters, Lietta Ferri, Milano: Il saggiatore.

Bellón Sánchez, Silvia (2015), “La violencia obstétrica desde los aportes de la crítica feminista y la biopolítica, Dilemata, 7(18), 93-111.

Belli, Laura (2013), "La violencia obstétrica: otra forma de violación a los derechos humanos", Revista Redbioética/UNESCO, 4, 1(7), 25-34.

www.elpartoesnuestro.es/sites/default/files/recu rsos/documents/declaracion_interovo.pdf 
Camacaro Cuevas, Marbella, (2012), La obstetricia desnuda. Una roptura epistemica. Caracas: Universidad de Carabobo.

Castrillo, Belén (2016), "Dime quién lo define $y$ te diré si es violento. Reflexiones sobre la violencia obstétrica", Sexualidad, Salud y Sociedad, 24, 43-68.

Castro, Roberto; Bronfam, Mario (1993), "Teoría Feminista y Sociología Médica: Bases para una Discusión", Cad. Saúde Públ, 9(3), 375-394.

Castro, Roberto; Erviti, Joaquina (2003), "Violation of reproductive rights during hospital births in Mexico", Health and Human Rights, 7(1), 91-110.

Castro, Roberto; Erviti, Joaquina (2015), Sociología de la práctica médica autoritaria. Violencia obstétrica, anticoncepción inducida y derechos reproductivos. Cuernavaca: CRIMUNAM.

Chiarotti, Susana; García Jurado, Mariana; Aucía, Analía, Armichiardi, Susana (2003), Con Todo al Aire 1. Reporte de derechos humanos sobre atención en salud reproductiva en hospitales públicos. Buenos Aires: INSGENAR, CLADEM.

Chiarotti, Susana; Schuster, Gloria; Armichiardi, Susana (2008), Con todo al aire 2. Reporte de Derechos humanos sobre atención en salud reproductiva en Hospitales públicos. Buenos Aires: INSGENAR, CLADEM.

Davis-Floyd, Robbie (1992), Birth as an American Rite of Passage. Barkley: University of California Press.
Davis-Floyd, Robbie; Sargent, Caroline (eds.) (1997), Childbirth and Authoritative Knowledge. CrossCultural Perspectives. Barkley: University of California Press.

Diniz Simone, Grilo; Salgado de Oliveira, Heloisa; Andrezzo de Aguiar, Halana Faria; Carvalho Cardin Galdino, Paula; Carvalho Albuquerque Cavalcanti, Priscila; Aguiar de Azevedo, Cláudia; Niy Yoshie, Denise (2014), "Abuse and disrespect in childbirth care as a public health issue in Brazil: origins, definitions, impacts on maternal health, and proposals for its prevention", Journal of Human Growth and Development. 25(3): 377-384. Doi: http://dx.doi.org/10.7322/jhgd.106080.

Foucault, Michel (1969), Nascita della clinica. Il ruolo della medicina nella costituzione delle scienze umane. Trad. Alessandro Fontana, Torino: Einaudi.

Foucault, Michel (1976), Sorvegliare e punire. Nascita della prigione. Trad. Alcesti Tarchetti, Torino: Einaudi.

Grupo de Información en Reproducción Elegida (2015), "Violencia Obstétrica", Niñas y mujeres sin justicia. Derechos reproductivos en México, México: GIRE.

Jordan, Brigitte (1997), "Authoritative knowledge and its construction", in Davis-Floyd Robby; Sargent, Caroline. (eds.) Childbirth and Authoritative Knowledge. Cross-Cultural Perspectives. Barkley: University of California Press, 55-88.

Las Casildas (2015), Observatorio de Violencia Obstétrica. Informe final. Buenos Aires: Las Casildas. 
Lock, Margaret; Nguyen, Vinh-Kim (2010), An Anthropology of Biomedicine. Oxford: Wiley-Blackwell.

Magnone Alemán, Natalia (2010), "Derechos sexuales y reproductivos en tensión: intervencionismo y violencia obstétrica", X Jornadas de Investigación de la Facultad de Ciencias Sociales, Montevideo: Universidad de la República.

Menéndez, Edoardo (2009), De sujetos, saberes y estructuras:Introducción al enfoque relacional en el estudio de la salud colectiva. Buenos Aires: Lugar Editorial.

Ministerio de Salud, (2015). Segundo Informe Nacional de Relevamiento epidemiológico (2015). Buenos Aires: Ministerio de Salud de la Nación, Organización Panamericana de Salud.

Olivier de Sardan, Jean Pierre (2005), Anthropology and Development: Understanding Contemporary Social Change. New York: Zed Books.

Perez De Gregorio, Rogélio (2010). "Obstetric violence: A new legal term introduced in Venezuela", International Journal of Gynaecology and Obstetrics. The Official Organ of the International Federation of Gynaecology and Obstetrics, 111(3), 201-202.

Sadler, Michelle; Santos, Mario; Ruíz Berdún, Dolores; Rojas Leiva, Gonzales; Skoko, Elena; Gillen Patricia, Clausen, Aaroe Jette (2016), "Moving beyond disrespect and abuse: Addressing the structural dimensions of obstetric violence", Reproductive Health Matters, 24(47), 47-55.

Terán, Pablo; Castellanos, Carlos; Gonzáles, Blanco Mireya, Ramos Damarys (2013), "Violencia obstétrica: percepción de las usuarias", Rev Obstet Ginecol Venez., 73(3), 171-180.

Valdez-Santiago, Rosario; HidalgoSolórzano, Elisa; Mojarro-Iñiguez, Mariana; Arenas-Monreal, Luz María (2013), "Nueva evidencia a un viejo problema: el abuso de las mujeres en las salas de parto", Revista Conamed, 18(1), 14-20.

World Health Organization (1985). "Appropriate technology for Birth", The Lancet, 24, 436-437.

Villanueva Egan, Luis Alberto; Gutiérrez, María Ahuja; Valdez Santiago, Rosario; Lezana Fernández, Miguel Ángel (2016), "De qué hablamos cuando hablamos de violencia obstétrica?", Revista Conamed, 21, 7-25.

World Health Organization (1996), Care in normal Birth. A practical Guide, Reproductive health and research. Report of a Technical working group. Geneve: World Health Organization.

World Health Organization (2008), WHO European Strategic Approach for making safer. Improving maternal and perinatal health. Copenhagen: World Health Organization, Regional Office for Europe.

World Health Organization (2014). The prevention and elimination of disrespect and abuse during facility-based childbirth. Geneva: World Health Organization.

World Health Organization (2015), WHO Statement on Caesarean Section Rates. Geneva, World Health Organization. 